\title{
Prevalence, conservation and functional analysis of Yersinia and Escherichia CRISPR regions in clinical Pseudomonas aeruginosa isolates
}

\begin{abstract}
Correspondence
G. A. O'Toole

georgeo@Dartmouth.edu
\end{abstract}

Received 24 September 2010

Revised 11 November 2010

Accepted 12 November 2010

\author{
K. C. Cady, ${ }^{1}$ A. S. White, ${ }^{1}$ J. H. Hammond, ${ }^{1}$ M. D. Abendroth, ${ }^{1}$ \\ R. S. G. Karthikeyan, ${ }^{2}$ P. Lalitha, ${ }^{2}$ M. E. Zegans ${ }^{1,3}$ and G. A. O'Toole ${ }^{1}$ \\ ${ }^{1}$ Department of Microbiology and Immunology, Dartmouth Medical School, Hanover, NH 03755, \\ USA \\ ${ }^{2}$ Department of Ocular Microbiology, Aravind Eye Hospital, Madurai, India \\ ${ }^{3}$ Department of Surgery, Dartmouth Medical School, Lebanon, NH 03766, USA
}

\begin{abstract}
Here, we report the characterization of 122 Pseudomonas aeruginosa clinical isolates from three distinct geographical locations: Dartmouth Hitchcock Medical Center in New Hampshire, USA, the Charles T. Campbell Eye Microbiology Lab at the University of Pittsburgh Medical Center, USA, and the Aravind Eye Hospital in Madurai, India. We identified and located clustered regularly interspaced short palindromic repeats (CRISPR) in 45/122 clinical isolates and sequenced these CRISPR, finding that Yersinia subtype CRISPR regions (33\%) were more prevalent than the Escherichia CRISPR region subtype (6\%) in these $P$. aeruginosa clinical isolates. Further, we observed 132 unique spacers from these 45 CRISPR that are $100 \%$ identical to prophages or sequenced temperate bacteriophage capable of becoming prophages. Most intriguingly, all of these 132 viral spacers matched to temperate bacteriophage/prophages capable of inserting into the host chromosome, but not to extrachromosomally replicating lytic $P$. aeruginosa bacteriophage. We next assessed the ability of the more prevalent Yersinia subtype CRISPR regions to mediate resistance to bacteriophage infection or lysogeny by deleting the entire CRISPR region from sequenced strain UCBPP-PA14 and six clinical isolates. We found no change in CRISPR-mediated resistance to bacteriophage infection or lysogeny rate even for CRISPR with spacers $100 \%$ identical to a region of the infecting bacteriophage. Lastly, to show these CRISPR and cas genes were expressed and functional, we demonstrated production of small CRISPR RNAs. This work provides both the first examination to our knowledge of CRISPR regions within clinical $P$. aeruginosa isolates and a collection of defined CRISPR-positive and -negative strains for further CRISPR and cas gene studies.
\end{abstract}

\section{INTRODUCTION}

The ability of mobile genetic elements to alter the fitness and/or phenotype of bacteria has been well established (Brüssow et al., 2004). Recent studies in members of the genera Pseudomonas, Streptococcus and Staphylococcus have demonstrated the importance of clustered regularly interspaced short palindromic repeats (CRISPR) in the interaction of bacteria and mobile genetic elements such as plasmids and bacteriophage (Barrangou et al., 2007; Marraffini \& Sontheimer, 2008; Zegans et al., 2009).

Abbreviations: CRISPR, clustered regularly interspaced short palindromic repeats; crRNA, CRISPR RNA; DHMC, Dartmouth Hitchcock Medical Center; EOP, efficiency of plaquing; PAM, proto-spacerassociated motifs.

A supplementary figure and five supplementary tables are available with the online version of this paper.
CRISPR are found in 39 and $88 \%$ of sequenced bacterial and archaeal genomes, respectively (van der Oost et al., 2009). Previous studies have shown that CRISPR regions have been categorized into eight distinct subtypes (Escherichia, Yersinia, Neisseria, Desulfovibrio, Thermotoga, Haloarcula, Apern and Mycobacterium) that are widely distributed in bacteria and archaea (Haft et al., 2005). CRISPR subtypes vary in both the sequence and length of their identical repeats as well as their repertoire of CRISPR associated (cas) genes (Haft et al., 2005). Many CRISPR and their cognate cas genes are found in the same genomic location, termed a CRISPR region (Zegans et al., 2009). Socalled general cas genes are found in multiple CRISPR region subtypes (Haft et al., 2005). Only one cas gene is found in all CRISPR region subtypes, namely the casl gene (Haft et al., 2005). However, each of these regions also harbours subtype-specific CRISPR-associated genes abbreviated $c s x$, 
where $x$ is the first letter of the subtype (example: csy for the Yersinia subtype) (Haft et al., 2005). Most cas genes are not shared between CRISPR region subtypes, calling into question the ability of different CRISPR regions to function similarly (Haft et al., 2005).

Previous studies have implicated CRISPR in the resistance to lytic bacteriophage and plasmids (Barrangou et al., 2007; Marraffini \& Sontheimer, 2008). However, native resistance to bacteriophage and plasmids has only been observed in Streptococcus thermophilus and Staphylococcus epidermidis, respectively (Barrangou et al., 2007; Brouns et al., 2008; Marraffini \& Sontheimer, 2008). While many bioinformatic studies have been published which match CRISPR spacer content to bacteriophage genomes (DíezVillaseñor et al., 2010; Held \& Whitaker, 2009; Shah et al., 2009; Vestergaard et al., 2008), few studies have attempted to test the ability of these native CRISPR regions to mediate functional bacteriophage-host interactions.

Here, we report the prevalence, location and sequence of CRISPR regions from Pseudomonas aeruginosa clinical isolates, thus providing a library of $P$. aeruginosa spacers matching sequenced bacteriophages. We also report the host range and contribution to resistance of these CRISPR to sequenced $P$. aeruginosa bacteriophage. Finally, we show that there is no evidence supporting a role for the Yersinia subtype CRISPR in mediating native resistance to bacteriophage infection or lysogeny of $P$. aeruginosa.

\section{METHODS}

Bacterial and phage culture conditions. Bacterial strains, bacteriophage strains, plasmids and primers used in this study are listed in Supplementary Tables S1 and S2 (available with the online version of this paper). P. aeruginosa and Escherichia coli strains were routinely cultured in lysogeny broth (LB) at $37^{\circ} \mathrm{C}$ (Bertani, 2004). The minimal medium M63 was supplemented with $\mathrm{MgSO}_{4}(1 \mathrm{mM})$ and arginine $(0.4 \%)$.

Growth media were supplemented with antibiotics at the following concentrations: ampicillin (Ap), $150 \mu \mathrm{g} \mathrm{ml}^{-1}$ (E. coli); gentamicin $(\mathrm{Gm}), 10 \mu \mathrm{g} \mathrm{ml}^{-1}$ (E. coli) and $50 \mu \mathrm{g} \mathrm{ml}^{-1}$ (P. aeruginosa); carbenacillin $50 \mu \mathrm{g} \mathrm{ml}^{-1}$ (E. coli) and $500 \mu \mathrm{g} \mathrm{ml}^{-1}$ (P. aeruginosa).

Deletion of $\boldsymbol{P}$. aeruginosa clinical isolate Yersinia subtype CRISPR regions. Regions of sequence identity upstream and downstream of the UCBPP-PA14 CRISPR region were amplified and cloned into a pMQ30 vector, using Saccharomyces cerevisiae recombineering as previously performed (Shanks et al., 2006). Clinical $P$. aeruginosa isolates harbouring Yersinia subtype CRISPR regions were spot mated with $E$. coli S17 harbouring the suicide pMQ30- $\Delta$ CR plasmid. Transformants were selected on gentamicin and nalidixic acid. Meridiploids were counter-selected on $5 \%$ sucrose before being tested for the clean deletion of the entire CRISPR region by PCR. Deletions were confirmed with primers $c s y 3$ amp For, csy3 amp Rev, $\Delta$ CR Check For(70) and $\Delta$ CR Check Rev(70). $\Delta$ CR Check For(70) and $\Delta$ CR Check $\operatorname{Rev}(70)$ are located external to the regions of DNA sequence homology found on plasmid pMQ30- $\Delta C R$ (Supplementary Table S2). Primers csy3 amp For and csy3 amp Rev were used to amplify csy3, providing both a positive and negative control for each strain (Supplementary Table S2).
Northern blot analysis. Northern blot assays were performed to determine the ability of $P$. aeruginosa strains to functionally produce small CRISPR RNAs (crRNAs) under laboratory conditions. Cultures grown overnight in LB medium were pelleted and resuspended in $100 \mu \mathrm{l} 400 \mu \mathrm{g}$ lysozyme $\mathrm{ml}^{-1}$ in TE buffer for 3-5 min at room temperature. Following lysozyme-mediated cell lysis, total RNA was purified using the mirVana miRNA isolation kit (Ambion), essentially following the manufacturer's instructions for total RNA extraction. Primers Northern Marker/probe For and Northern Marker/probe Rev2 were utilized in PCRs to synthesize a probe comprising the last four spacers and three repeats of CRISPR2. The random primed DNA labelling kit (Roche) and $\left[\alpha-{ }^{32} \mathrm{P}\right] \mathrm{dCTP}[3000 \mathrm{Ci}$ $\mathrm{mmol}^{-1}\left(111 \mathrm{TBq} \mathrm{mmol}^{-1}\right)$; PerkinElmer] were utilized according to the manufacturer's specification to radioactively label the probe. mirVana-suggested sRNA prehybridization, hybridization and wash solutions were utilized at 65,37 and $37{ }^{\circ} \mathrm{C}$, respectively. These conditions were chosen because they allowed our probe, containing four CRISPR spacers and three CRISPR repeats, to hybridize with the conserved repeat sequence of all Yersinia subtype CRISPRderived small crRNAs regardless of spacer content. Following the third 15 min wash, membranes were covered with plastic wrap and exposed to a storage phosphor screen (GE Healthcare) overnight. After exposure, phosphor screens were analysed on a Storm 860 (Molecular Devices) and processed using Image Quant software v5.2 (Molecular Devices).

Measurement of efficiency of plaquing (EOP). Bacteriophage lysates were prepared by filter-sterilizing overnight LB-grown cultures of either P. aeruginosa strains UCBPP-PA14 or PAO1 lysogenized by the given temperate bacteriophage. Top agar lawns of CRISPRregion-positive and -negative strains were prepared as described previously (Zegans et al., 2009), and spotted with serial dilutions of three independent bacteriophage preparations. The EOP was determined by dividing the titre of a single bacteriophage preparation on the CRISPR region deletion strain by the titre obtained on the cognate CRISPR-region-positive strain. Standard deviations were obtained by comparison of the EOP obtained for three independent bacteriophage preparations.

Measurement of lysogeny frequency. Similar to EOP measurements, bacteriophage lysates were prepared by filter-sterilizing overnight LB-grown cultures of $P$. aeruginosa strain UCBPP-PA14 lysogenized by either DMS3 or MP22. Microcentrifuge tubes containing $500 \mu \mathrm{l}$ of sterile LB medium were inoculated with $10 \mu \mathrm{l}$ of an overnight culture of either wild-type $P$. aeruginosa UCBPPPA14 or the $\triangle \mathrm{CR}$ mutant. This dilute bacterial culture was then inoculated with $10 \mu \mathrm{l}$ purified bacteriophage $\left(10^{7}\right.$ p.f.u. $\left.\mathrm{ml}^{-1}\right)$ and incubated at $37{ }^{\circ} \mathrm{C}$ for $25 \mathrm{~min}$. Following incubation, cultures were washed twice with fresh LB medium to remove all remaining infectious bacteriophage particles. Serial dilutions were performed, plated on LB agar and incubated overnight at $37{ }^{\circ} \mathrm{C}$. Cross-streak test plates were prepared by dripping $30 \mu$ l bacteriophage lysate down the centre of an LB agar plate (Davis et al., 1980). Single colonies were picked from plates and streaked perpendicularly across the central bacteriophage lysate line of the cross-streak test plates. Bacteria that had become lysogens were immune to super-infection by the same bacteriophage; however, non-infected bacteria grew poorly after passing through the concentrated bacteriophage lysate (Davis et al., 1980). Three independent assays were performed for each strain, with 50 colonies cross-streaked for every experiment (150 total colonies per strain). Statistical analysis was performed using the Fischer exact test.

CRISPR region sequencing and annotation. Clinical isolates harbouring Yersinia subtype CRISPR regions were amplified by PCR with primers Yersinia subtype CR amp For(70) and Yersinia subtype CR amp Rev(70) using Phusion polymerase (New England Biolabs, Beverley, MA). The resulting $\sim 12 \mathrm{~kb}$ product was gel purified 
(Qiagen), denatured at $96{ }^{\circ} \mathrm{C}$ for $5 \mathrm{~min}$, and sequenced on a ABI 3730 DNA analyser sequencer using primers Yersinia subtype CRISPR1 SEQ FOR(70), Yersinia subtype CRISPR1 SEQ REV(70), Yersinia subtype CRISPR2 SEQ FOR(70), and Yersinia subtype CRISPR2 SEQ REV(70). Sequencing gaps were closed using unique primers proximal or distal to the sequencing gap (primer sequences not shown). Annotation of each CRISPR1 and 2 was performed manually and entered into the redundant spacer library (Supplementary Table S3, available with the online version of this paper).

Clinical isolates harbouring Escherichia subtype CRISPR regions were treated similarly except primers Escherichia subtype CR amp For(72) and Escherichia subtype CR amp $\operatorname{Rev}(72)$ were used to amplify the $\sim 10 \mathrm{~kb}$ region and primers Escherichia subtype CRISPR1 SEQ FOR(72), Escherichia subtype CRISPR1 SEQ REV(69), Escherichia subtype CRISPR2 SEQ FOR(70), and Escherichia subtype CRISPR2 SEQ REV(72) were utilized in sequencing reactions.

Redundant and non-redundant spacer libraries. The spacer content of each strain was entered into a redundant spacer library (Supplementary Table S3). We also created a non-redundant library in which each unique spacer was represented in the database only once (Supplementary Table S4, available with the online version of this paper). Each non-redundant spacer was then compared against the NCBI database using BLASTN to identify all $100 \%$ identical spacer targets using the program iSpacer (identify spacer).

iSpacer. iSpacer was created to increase the speed and accuracy at which large spacer libraries can be compared to the NCBI sequence database. iSpacer is written in the Python programming language, and functions on both PC and Mac platforms. Spacer libraries in FASTA format are opened in iSpacer, compared against the entire NCBI database at various stringency levels, and the resulting proto-spacer matches are exported into .txt and Microsoft Excel formatted files. The iSpacer program and supporting material is available free upon request from the corresponding author.

\section{RESULTS}

\section{Prevalence and location of Pseudomonas aeruginosa CRISPR regions}

We determined the prevalence of Yersinia and Escherichia subtype CRISPR regions in 122 clinical isolates of $P$. aeruginosa from three distinct geographical locations. Fifty isolates were obtained from Dartmouth Hitchcock Medical Center (DHMC) (Lebanon, NH, USA), 43 were from the Charles T. Campbell Eye Microbiology Lab (Pittsburgh, PA, USA), and 29 were from Aravind Eye Hospital (Madurai, Tamil Nadu, India). Isolates were obtained from $P$. aeruginosa sputum, urine, cystic fibrosis, and eye infections. Given the geographical and clinical diversity of this collection, it was likely these isolates have experienced a wide range of CRISPR-bacteriophage interactions.

To determine the prevalence of Yersinia and Escherichia subtype CRISPR regions, we amplified the subtype-specific cas genes csyl and cse3, respectively. Primers specific for csyl and cse 3 were verified on the only two sequenced $P$. aeruginosa strains harbouring CRISPR regions (UCBPPPA14 and PA2192). The conserved non-CRISPR/cas related gene pilG was utilized as a positive control for all
PCRs. Utilizing primers specific to $P$. aeruginosa strain UCBPP-PA14 csyl gene, we found that 40 of the 122 clinical isolates putatively harbour Yersinia subtype CRISPR regions (33\%). Campbell Eye Microbiology Lab samples had a slightly higher Yersinia subtype prevalence $(42 \%)$ than those from DHMC or Aravind Eye Hospital (22 and $38 \%$, respectively). Initially we believed that these CRISPR/cas regions would be randomly distributed in different locations throughout the clinical isolate genomes because CRISPR regions are thought to be highly mobile genomic regions (Chakraborty et al., 2010). However, all 40 Yersinia subtype CRISPR regions we mapped were located between homologues of the PA14_33290 and PA14_33370 ORFs.

Likewise, primers specific to the $P$. aeruginosa strain PA2192 cse3 gene led to the discovery of seven isolates harbouring Escherichia subtype regions (6\%). Escherichia subtype CRISPR regions were found in $10 \%$ of Aravind Eye Hospital isolates, while only 6 and $2 \%$ of DHMC and Campbell Eye Microbiology Lab samples, respectively, harboured these regions. Similar to the Yersinia subtype, all Escherichia CRISPR regions were found in the same genomic location, between the PA14_48160 and PA14_ 48150 ORF.

Interestingly, sequenced $P$. aeruginosa strain PA2192 and three of the seven clinical isolates harbouring an Escherichia subtype CRISPR region also contain a Yersinia subtype region.

While we believe that this PCR-based analysis of these clinical isolates is thorough, we likely overlooked strongly degenerate Yersinia and Escherichia subtype CRISPR regions, as well as CRISPR regions of other subtypes.

\section{CRISPR region conservation and spacer sequence}

Sequencing of CRISPR regions was accomplished by amplification of the entire $\sim 12 \mathrm{~kb}$ Yersinia and $\sim 10 \mathrm{~kb}$ Escherichia CRISPR regions by PCR (Fig. 1a), followed by gel purification and sequencing of PCR products. Initially, both cas genes and CRISPR were sequenced, but it was quickly evident that cas sequence and organization were well conserved with previously sequenced $P$. aeruginosa strains UCBPP-PA14 and PA2192. Thus, the high conservation of cas gene sequences prompted us to focus on sequencing the more variable CRISPR sequences. Intriguingly, both Yersinia and Escherichia CRISPR regions have similar organizations. Both regions harbour two CRISPR with slightly different repeat sequences flanking a series of cas genes (Fig. 1b).

To analyse the spacer content of these strains, we sequenced each CRISPR and created a redundant spacer library with every spacer from all of the CRISPR-positive clinical isolates analysed (Supplementary Table S3). In order to follow the standard numbering system for CRISPR spacers presented in Supplementary Table S3, we made the assumption based 
(a)

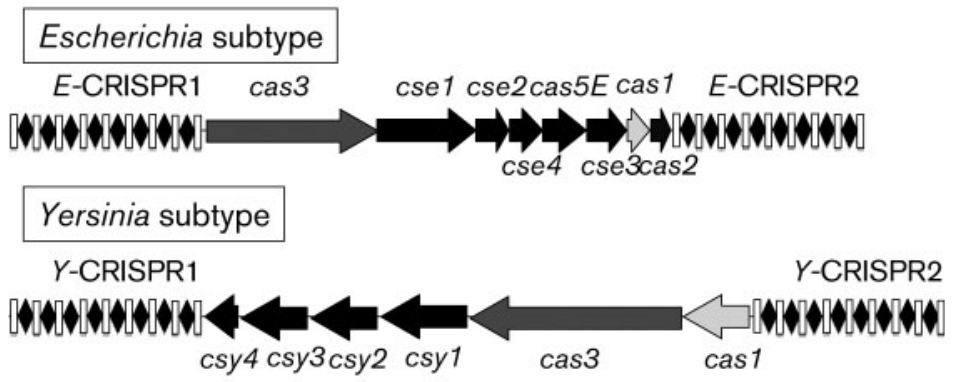

(b)

\begin{tabular}{|c|c|}
\hline CRISPR & Repeat sequence \\
\hline E-CRISPR1 & GTGTCCCCACGGGIGTGGGGATGAACCG \\
\hline E-CRISPR2 & GTGTCCCCACATGCGTGGGGATGAACCG \\
\hline$\gamma$-CRISPR1 & ПTСTTAGCTGCCTAIACGGCAGTGAAC \\
\hline$Y$-CRISPR2 & ПTாCTIAGCTGCCTACACGGCAGTGAAC \\
\hline
\end{tabular}

Fig. 1. Organization and conservation of $P$. aeruginosa CRISPR regions. (a) Organization of cas genes and flanking CRISPR are shown for the Escherichia subtype (top) and Yersinia subtype (bottom). Spacers are shown as diamonds and repeats as rectangles. Arrows indicate cas genes and their predicted direction of transcription. (b) Repeat sequences of the Yersinia and Escherichia subtypes (differences are underlined). on published studies (Barrangou et al., 2007; Deveau et al., 2008) that spacer acquisition is polar, taking place at one end of a given stretch of each CRISPR. Convention states that spacers are numbered starting from the terminus where new spacers are acquired (Deveau et al., 2008). The data used to support the numbering system presented in Supplementary Table S3 are presented in Supplementary Fig. S1 (available with the online version of this paper). It is worth noting that the most prevalent spacer repertoires for Yersinia and Escherichia subtype CRISPR are similar to the CRISPR spacer sequences found in $P$. aeruginosa strains UCBPPPA14 and 2192, respectively.

We also created a non-redundant library of all sequenced spacers, which is presented in Supplementary Table S4. This non-redundant spacer library contains 656 unique spacers, which were independently compared against the NCBI database. This comparison was performed using the newly developed iSpacer program, which can compare large lists of spacer sequences to the NCBI database using BLASTN, to determine regions of the bacterial and bacteriophage genomes that are identical to spacer sequences.

The sequences identified by using iSpacer analysis could be categorized into five groups, those spacers matching: (i) sequenced lysogenic $P$. aeruginosa bacteriophage $(n=65)$, (ii) prophages within sequenced $P$. aeruginosa clinical isolates $(n=95)$, (iii) transposons $(n=2)$, (iv) pathogenicity islands $(n=21)$, and (v) previously sequenced $P$. aeruginosa CRISPR regions (Supplementary Table S4).

Among the spacers that showed $100 \%$ identity to nonCRISPR sequences, 65 independent spacers were $100 \%$ identical to portions of 12 sequenced, well-characterized lysogenic $P$. aeruginosa bacteriophage. Fig. 2 illustrates the location of spacers matching eight representative sequenced bacteriophages: DMS3, MP22, D3112, D3, PAJU2, B3, ФCTX and F116 (Braid et al., 2004; Byrne \&
Kropinski, 2005; Heo et al., 2007; Kropinski, 2000; Nakayama et al., 1999; Uchiyama et al., 2009; Wang et al., 2004; Zegans et al., 2009). Further, 95 unique spacers were found to match uncharacterized prophages on the genomes of $P$. aeruginosa clinical isolates. These prophages were sequenced as part of bacterial genome sequencing projects or obtained from metagenomic studies of cystic fibrosis isolates (Hayden et al., 2008; Roy et al., 2010; Winstanley et al., 2009). Interestingly, a number of these spacers matched sequences of prophages linked to the increased virulence of the Liverpool epidemic strain LESB58 (Supplementary Table S5) (Winstanley et al., 2009). Only two spacers matched to transposons; oddly these spacers were also the only spacers that matched to non- $P$. aeruginosa sequences (these non-transposon matches were not included in Supplementary Table S4). Two spacers were also identical to pyocin gene fragments, which are vestigial bacteriophage tail fibres used by $P$. aeruginosa to puncture the membrane of competing organisms (Lee et al., 1999).

We also identified 21 unique spacers that matched to $\underline{P}$. aeruginosa genomic islands (PAGI). Great care was taken to differentiate spacer matches within putative lysogenic bacteriophage genomes from those matching PAGI. Twelve unique spacers were $100 \%$ homologous to PAGI6 , of which five also matched to the ФCTX genome, a finding consistent with previous reports detailing the sequence conservation between the mobile genomic island PAGI-6 and the cytotoxin-encoding bacteriophage ФCTX (Battle et al., 2009). PAGI-5 and the P. aeruginosa UCBPPPA14 pathogenicity island 1 (PAPI-1) are members of a large family of genomic islands closely related to large integrating $103.5 \mathrm{~kb}$ pKLC102-like plasmids first discovered in P. aeruginosa clone C strains (Battle et al., 2009). Nine unique spacers matched to PAGI-5-like elements, such as PAPI-1. 


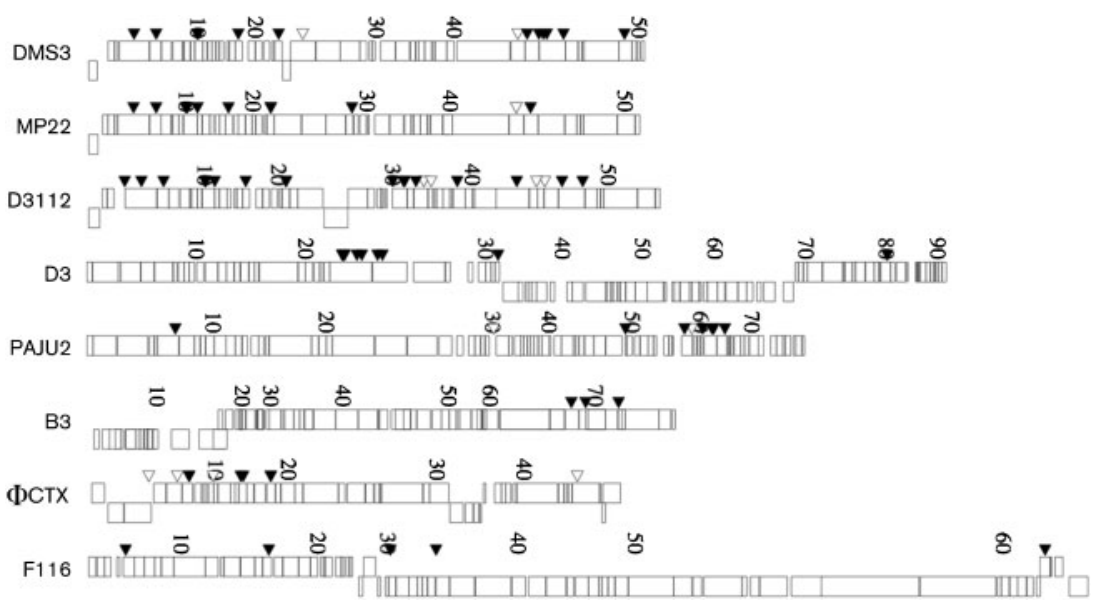

Fig. 2. Location of spacers $100 \%$ identical to common temperate $P$. aeruginosa bacteriophage. Boxes denote known or putative bacteriophage ORFs that have been published previously. Forward oriented ORFs are depicted above those ORFs that are divergently transcribed. Each arrow signifies the site of a proto-spacer sequence 100\% identical to a spacer identified in our analysis, with Yersinia subtype spacer matches shown as black triangles and Escherichia subtype spacer matche shown as open triangles.

\section{Proto-spacer matches and proto-spacer- associated motifs (PAMs)}

We analysed the flanking sequence of the putative protospacers corresponding to the unique spacers identified here (data not shown) and found conserved PAMs that were different between Yersinia and Escherichia subtypes. Yersinia subtypes contained a conserved GG dinucleotide immediately adjacent to their spacer, while Escherichia subtypes had a consensus CTT PAM. Both of these PAM motifs matches those previously discovered using spacer matches from many bacterial species containing the same CRISPR subtype (Mojica et al., 2009). Our large library of $100 \%$ identical spacer matches also showed that PAGI, prophages and previously sequenced bacteriophage all retained the same PAM sequence for both CRISPR subtypes (data not shown).

\section{Analysis of CRISPR-mediated resistance using EOP and lysogeny assays}

Previous studies implicated CRISPR and cas genes in resistance to lytic bacteriophages and to plasmids (Barrangou et al., 2007; Marraffini \& Sontheimer, 2008). To test the ability of the more prevalent Yersinia subtype CRISPR regions to mediate bacteriophage resistance, we identified clinical isolates with CRISPR regions harbouring one or more unique spacers $100 \%$ identical to sequenced bacteriophage. We then created mutant strains lacking the entire CRISPR region, and these deletion strains served as a control for non-CRISPR-mediated interactions. We assessed the resistance of each wild-type clinical isolate and its CRISPR deletion derivative to bacteriophage infection using an assay that measured the EOP.

Sequenced bacteriophage D3, DMS3, MP22 and F116 were utilized to infect clinical isolates harbouring spacers $100 \%$ identical to proto-spacer regions within the given bacteriophage. For most bacteriophages tested, we observed no ability to plaque on the clinical strains tested regardless of the presence or absence of the CRISPR region (Table 1). For those strains that could support plaquing by DMS3 and/or MP22, we found no evidence for CRISPRdependent resistance to bacteriophage as judged by a plaque assay (Table 1). That is, the EOP was approximately 1 in all experiments. These data indicate that for the strains and bacteriophage tested, CRISPR did not confer resistance to infection.

To determine if Yersinia subtype CRISPR regions harbouring $100 \%$ identical spacer matches to temperate bacteriophage impact the rate at which lysogeny occurs, we tested the frequency at which $P$. aeruginosa UCBPP-PA14 was lysogenized by bacteriophages MP22 and DMS3 in the presence or absence of the Yersinia subtype CRISPR region (harbouring one or more spacers $100 \%$ identical to these bacteriophage). We observed, by cross-streak test, that $8 \%$ of CRISPR-positive bacteria and $6 \%$ of $\Delta \mathrm{CR}$ bacteria became lysogenized by bacteriophage DMS3. Similarly, $2 \%$ of CRISPR-positive and $1.3 \%$ of $\triangle \mathrm{CR}$ bacteria were lysogenized by the MP22 bacteriophage. Both of these lysogeny rates were not found to be significantly different

Table 1. Measuring EOP of clinical isolates

$\mathrm{EOP}$ is given as mean $\pm \mathrm{SD}$. EOP of 1 indicates no change in resistance. NCR, Non-CRISPR-mediated resistance, assessed by the lack of plaque formation in the CRISPR-negative clinical isolate.

\begin{tabular}{|lcc|}
\hline Strain & Bacteriophage & EOP \\
\hline PA14 $\Delta$ CR & DMS3 & $1.06 \pm 0.2$ \\
& MP22 & $1.95 \pm 1.0$ \\
SMC4485 $\Delta$ CR & F116 & NCR \\
& DMS3 & $1.53 \pm 0.5$ \\
MMC4515 $\Delta$ CR & MP22 & $2.10 \pm 1.6$ \\
& F116 & NCR \\
SMC4513 $\Delta$ CR & MP22 & $0.92 \pm 0.3$ \\
SMC4384 $\Delta$ CR & F116 & NCR \\
SMC4502 $\Delta$ CR & DMS3 & NCR \\
SMC4504 $\Delta$ CR & D3 & NCR \\
& DMS3 & NCR \\
& F116 & NCR \\
\hline
\end{tabular}


between CRISPR-positive and -negative strains using the Fisher exact test $(P=0.65$ and $P=1$, respectively).

\section{crRNA production from clinical isolates}

We next assessed whether the CRISPR transcript in the clinical isolates described above were processed into small RNAs. From the presence of crRNA we could infer that the cas genes were expressed and each product(s) was functional (Haurwitz et al., 2010). Northern blot analysis showed that each strain produced crRNAs, and the production of these crRNAs was dependent on the presence of the CRISPR region (Fig. 3). These data indicate that Yersinia subtype $P$. aeruginosa CRISPR regions are expressed and functionally able to produce crRNAs under our experimental conditions.

\section{DISCUSSION}

This work demonstrates that $P$. aeruginosa Yersinia subtype CRISPR did not mediate native resistance to bacteriophages, even when the bacteriophages contained sequences that were $100 \%$ identical to spacers within the CRISPR. Widely accepted CRISPR dogma would suggest that when challenged by the bacteriophage with sequences $100 \%$ identical to spacers, these CRISPR regions should respond by mediating resistance to the given bacteriophage (Barrangou et al., 2007). To test this model with the Yersinia subtype CRISPR, we analysed multiple clinical isolates harbouring a diverse range of spacers against a collection of sequenced $P$. aeruginosa bacteriophage. However, we found that of the seven strains tested, none mediated CRISPR-dependent resistance to bacteriophage. Further, $P$. aeruginosa strain UCBPP-PA14, which harbours spacers $100 \%$ identical to bacteriophage MP22 and DMS3, showed no difference in the rate of lysogeny for either of these temperate bacteriophage in the wild-type versus $\Delta \mathrm{CR}$ strains. Thus, two of the previously described host-bacteriophage interactions mediated by CRISPR (Barrangou et al., 2007; Edgar \& Qimron, 2010) are not

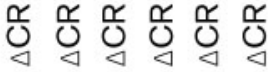

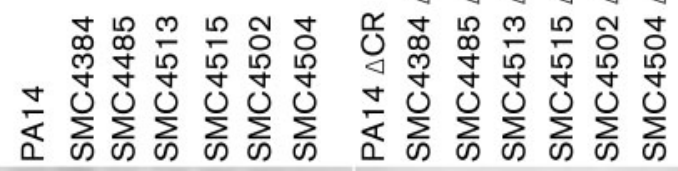

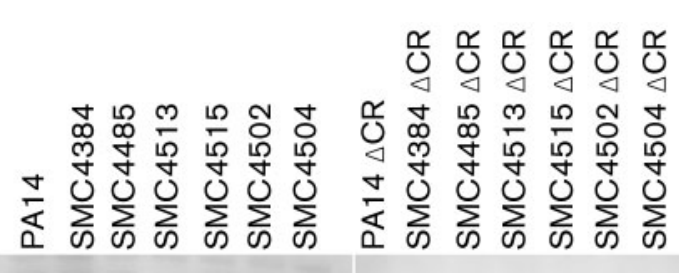

crRNA

Fig. 3. Characterization of CRISPR-positive and -negative $P$. aeruginosa clinical isolates. Northern blot analysis of small crRNA production for the wild-type lab strain and six clinical isolates (left) and their $\triangle$ CRISPR derivatives (right). In this experiment, the three spacers and two repeats closest to the cas 1 gene of CRISPR2 of $P$. aeruginosa UCBPP-PA14 were used as the probe. impacted by the Yersinia subtype CRISPR region. The observation that Yersinia subtype CRISPR regions produced small crRNA in all of the strains tested demonstrates that these regions are functional under our laboratory conditions. Thus, our findings argue against the conclusion that these regions are 'silent', as has been suggested for CRISPR regions within E. coli (Díez-Villaseñor et al., 2010; Pougach et al., 2010; Westra et al., 2010).

While previous studies of other CRISPR region subtypes have reported the importance of short PAM motifs adjacent to the bacteriophage genomic sequence matching to CRISPR region spacers (proto-spacers) (Deveau et al., 2008), no study has demonstrated their requirement in Yersinia subtype CRISPR-mediated interactions. Our analysis of bacteriophage MP22 demonstrated no CRISPR-dependent alteration in EOP or frequency of lysogeny when the bacterial host strain harbours a spacer $100 \%$ identical to a bacteriophage proto-spacer with a conserved PAM. Unfortunately, in this work we did not observe any CRISPR-dependent alteration in lysogeny rate or bacteriophage resistance, and so cannot comment on the requirement of the PAM motifs in our studies.

Our analysis showed that the Yersinia and Escherichia subtype CRISPR regions of $P$. aeruginosa clinical isolates harbour spacers against mobile genetic elements that integrate within the host chromosome. All 132 unique spacers $100 \%$ identical to viral sequences match to either well-studied temperate $P$. aeruginosa bacteriophage capable of integrating into the host chromosome or prophages within the genome of $P$. aeruginosa clinical isolates (Braid et al., 2004; Byrne \& Kropinski, 2005; Heo et al., 2007; Kropinski, 2000; Lee et al., 1999; Nakayama et al., 1999; Roy et al., 2010; Uchiyama et al., 2009; Wang et al., 2004; Winstanley et al., 2009; Zegans et al., 2009). We also identified additional spacer sequences that match PAGI and transposons. Spacers matching to extrachromosomally replicating bacteriophage, which is typical of lytic bacteriophage, and plasmids are conspicuously absent from our dataset. Our data are also consistent with a previous report describing Escherichia and Yersinia subtype CRISPR regions which stated that of 100 E. coli strains tested, $65.4 \%$ of bacteriophage-targeting spacers corresponded to a prophage within the E. coli isolates E24377A and SE11 (Díez-Villaseñor et al., 2010). This seemingly preferential targeting of prophages coupled with spacers matching to PAGI and transposons raises the possibility that the natural role of these CRISPR regions is in the interaction with chromosomally integrated mobile genetic elements rather than in mediating defence against extra-chromosomal infecting bacteriophages and plasmids.

How different CRISPR region subtypes interact with protospacer targets is an area of active research and speculation. However, when one considers that casl, which is thought to insert new spacers, is the only cas gene found in all CRISPR subtypes it is perhaps less surprising that these regions may have been co-opted for varied roles in 
different bacteria. An example of this diversity of action is that the Neisseria subtype CRISPR region of Streptococcus thermophilus is believed to interact with the DNA of invading bacteriophage, while the Thermotoga subtype of Pyrococcus furiosus has been shown to interact directly with RNA (Hale et al., 2009).

We found 41 unique clinical $P$. aeruginosa CRISPR spacers $100 \%$ identical to known horizontally transferable virulence factors, as well as 112 spacers identical to other putative virulence factors within PAGI, prophages and lysogenic bacteriophage. Transposons, prophages and genomic islands are thought to be responsible for the majority of strain diversity in P. aeruginosa (Battle et al., 2008, 2009; Winstanley et al., 2009). This type of genomic diversity is perhaps best exemplified by the Liverpool epidemic strain of P. aeruginosa (strain LESB58). Prior to this LESB58 study, it was assumed that most $P$. aeruginosa strains had very similar genomic content and few unique virulence factors that would make one strain considerably more virulent than another. However, unbiased signature-tagged mutagenesis coupled with genome sequencing revealed that many of the strain-specific genomic islands and prophages within strain LESB58 were critical to the increased virulence of this strain of $P$. aeruginosa (Winstanley et al., 2009). Strikingly, three of the four prophage genomes implicated in contributing to LESB58 virulence in a chronic rat lung model of infection were targeted by one or more unique spacers within our library. Similarly, we identified many unique spacers targeting the previously identified virulence-modulating lysogenic $P$. aeruginosa bacteriophages D3 and ФCTX. In addition, nine unique spacers targeted PAGI-5/PAPI-I-like elements, which have been shown to contribute to the virulence of $P$. aeruginosa clinical isolate PSE9 within the mouse model of acute pneumonia (Battle et al., 2008). The observation that human clinical isolates of $P$. aeruginosa have acquired and retained spacers $100 \%$ identical to known and putative chromosomal virulence factors raises the intriguing hypothesis that these CRISPR and cas genes are mediating a clinically important interaction between the bacterial host and mobile genetic elements.

\section{ACKNOWLEDGEMENTS}

We thank Rory T. Allar, Dae Gon Ha and Thomas Hampton for preliminary experiments or statistical analysis. Bacteriophage or bacteria strains were contributed by the Cho lab (Sogang University, South Korea), Collection de phages Félix d'Herelle (Université Laval, France), the Maillard lab (Cardiff University, UK), the Lory Lab (Harvard Medical School, USA) and the Charles T. Campbell Eye Microbiology Lab (University of Pittsburgh, USA). This work was supported by a National Institutes of Health predoctoral fellowship to K. C. C. (T32 AI007519), the Dartmouth Dickey Center, and National Institutes of Health grants R01AI083256 and R21AI068662 to G. A. O.

\section{REFERENCES}

Barrangou, R., Fremaux, C., Deveau, H., Richards, M., Boyaval, P., Moineau, S., Romero, D. \& Horvath, P. (2007). CRISPR provides acquired resistance against viruses in prokaryotes. Science 315, 17091712.

Battle, S. E., Meyer, F., Rello, J., Kung, V. \& Hauser, A. (2008). Hybrid pathogenicity island PAGI-5 contributes to the highly virulent phenotype of a Pseudomonas aeruginosa isolate in mammals. J Bacteriol 190, 7130-7140.

Battle, S. E., Rello, J. \& Hauser, A. (2009). Genomic islands of Pseudomonas aeruginosa. FEMS Microbiol Lett 290, 70-78.

Bertani, G. (2004). Lysogeny at mid-twentieth century: P1, P2, and other experimental systems. J Bacteriol 186, 595-600.

Braid, M. D., Silhavy, J., Kitts, C., Cano, R. \& Howe, M. (2004). Complete genomic sequence of bacteriophage B3, a Mu-like phage of Pseudomonas aeruginosa. J Bacteriol 186, 6560-6574.

Brouns, S. J., Jore, M. M., Lundgren, M., Westra, E. R., Slijkhuis, R. J., Snijders, A. P., Dickman, M. J., Makarova, K. S., Koonin, E. V. \& van der Oost, J. (2008). Small CRISPR RNAs guide antiviral defense in prokaryotes. Science 321, 960-964.

Brüssow, H., Canchaya, C. \& Hardt, W. (2004). Phages and the evolution of bacterial pathogens: from genomic rearrangements to lysogenic conversion. Microbiol Mol Biol Rev 68, 560-602.

Byrne, M. \& Kropinski, A. (2005). The genome of the Pseudomonas aeruginosa generalized transducing bacteriophage F116. Gene 346, 187-194.

Chakraborty, S., Snijders, A., Chakravorty, R., Ahmed, M., Tarek, A. \& Hossain, M. (2010). Comparative network clustering of direct repeats (DRs) and cas genes confirms the possibility of the horizontal transfer of CRISPR locus among bacteria. Mol Phylogenet Evol 56, 878-887.

Davis, R., Botstein, D. \& Roth, J. (1980). Advanced Bacterial Genetics. Cold Spring Harbor, NY: Cold Spring Harbor Laboratory.

Deveau, H., Barrangou, R., Garneau, J., Labonté, J., Fremaux, C., Boyaval, P., Romero, D., Horvath, P. \& Moineau, S. (2008). Phage response to CRISPR-encoded resistance in Streptococcus thermophilus. J Bacteriol 190, 1390-1400.

Díez-Villaseñor, C., Almendros, C., García-Martínez, J. \& Mojica, F. (2010). Diversity of CRISPR loci in Escherichia coli. Microbiology 156, 1351-1361.

Edgar, R. \& Qimron, U. (2010). The Escherichia coli CRISPR system protects from $\lambda$ lysogenization, lysogens, and prophage induction. J Bacteriol 192, 6291-6294.

Haft, D. H., Selengut, J., Mongodin, E. \& Nelson, K. (2005). A guild of 45 CRISPR-associated (Cas) protein families and multiple CRISPR/ Cas subtypes exist in prokaryotic genomes. PLOS Comput Biol 1, e60.

Hale, C. R., Zhao, P., Olson, S., Duff, M., Graveley, B., Wells, L., Terns, R. \& Terns, M. (2009). RNA-guided RNA cleavage by a CRISPR RNA-Cas protein complex. Cell 139, 945-956.

Haurwitz, R. E., Jinek, M., Wiedenheft, B., Zhou, K. \& Doudna, J. (2010). Sequence- and structure-specific RNA processing by a CRISPR endonuclease. Science 329, 1355-1358.

Hayden, H. S., Gillett, W., Saenphimmachak, C., Lim, R., Zhou, Y., Jacobs, M. A., Chang, J., Rohmer, L., D'Argenio, D. A. \& other authors (2008). Large-insert genome analysis technology detects structural variation in Pseudomonas aeruginosa clinical strains from cystic fibrosis patients. Genomics 91, 530-537.

Held, N. L. \& Whitaker, R. (2009). Viral biogeography revealed by signatures in Sulfolobus islandicus genomes. Environ Microbiol 11, 457-466.

Heo, Y. J., Chung, I., Choi, K., Lau, G. \& Cho, Y. (2007). Genome sequence comparison and superinfection between two related Pseudomonas aeruginosa phages, D3112 and MP22. Microbiology 153, 2885-2895. 
Kropinski, A. M. (2000). Sequence of the genome of the temperate, serotype-converting, Pseudomonas aeruginosa bacteriophage D3. J Bacteriol 182, 6066-6074.

Lee, F. K., Dudas, K., Hanson, J., Nelson, M., LoVerde, P. \& Apicella, M. (1999). The R-type pyocin of Pseudomonas aeruginosa $\mathrm{C}$ is a bacteriophage tail-like particle that contains single-stranded DNA. Infect Immun 67, 717-725.

Marraffini, L. A. \& Sontheimer, E. (2008). CRISPR interference limits horizontal gene transfer in staphylococci by targeting DNA. Science 322, 1843-1845.

Mojica, F. J., Díez-Villaseñor, C., García-Martínez, J. \& Almendros, C. (2009). Short motif sequences determine the targets of the prokaryotic CRISPR defence system. Microbiology 155, 733-740.

Nakayama, K., Kanaya, S., Ohnishi, M., Terawaki, Y. \& Hayashi, T. (1999). The complete nucleotide sequence of phi CTX, a cytotoxinconverting phage of Pseudomonas aeruginosa: implications for phage evolution and horizontal gene transfer via bacteriophages. Mol Microbiol 31, 399-419.

Pougach, K., Semenova, E., Bogdanova, E., Datsenko, K., Djordjevic, M., Wanner, B. \& Severinov, K. (2010). Transcription, processing and function of CRISPR cassettes in Escherichia coli. Mol Microbiol 77, 1367-1379.

Roy, P. H., Tetu, S., Larouche, A., Elbourne, L., Tremblay, S., Ren, Q., Dodson, R., Harkins, D., Shay, R. \& other authors (2010). Complete genome sequence of the multiresistant taxonomic outlier Pseudomonas aeruginosa PA7. PLoS ONE 5, e8842.

Shah, S. A., Hansen, N. \& Garrett, R. (2009). Distribution of CRISPR spacer matches in viruses and plasmids of crenarchaeal acidothermophiles and implications for their inhibitory mechanism. Biochem Soc Trans 37, 23-28.

Shanks, R. M., Caiazza, N., Hinsa, S., Toutain, C. \& O'Toole, G. (2006). Saccharomyces cerevisiae-based molecular tool kit for manipulation of genes from gram-negative bacteria. Appl Environ Microbiol 72, 5027-5036.

Uchiyama, J., Rashel, M., Matsumoto, T., Sumiyama, Y., Wakiguchi, H. \& Matsuzaki, S. (2009). Characteristics of a novel Pseudomonas aeruginosa bacteriophage, PAJU2, which is genetically related to bacteriophage D3. Virus Res 139, 131-134.

van der Oost, J., Jore, M., Westra, E., Lundgren, M. \& Brouns, S. (2009). CRISPR-based adaptive and heritable immunity in prokaryotes. Trends Biochem Sci 34, 401-407.

Vestergaard, G., Shah, S., Bize, A., Reitberger, W., Reuter, M., Phan, H., Briegel, A., Rachel, R., Garrett, R. A. \& Prangishvili, D. (2008). Stygiolobus rod-shaped virus and the interplay of crenarchaeal rudiviruses with the CRISPR antiviral system. J Bacteriol 190, 6837-6845.

Wang, P. W., Chu, L. \& Guttman, D. (2004). Complete sequence and evolutionary genomic analysis of the Pseudomonas aeruginosa transposable bacteriophage D3112. J Bacteriol 186, 400-410.

Westra, E. R., Pul, U., Heidrich, N., Jore, M. M., Lundgren, M., Stratmann, T., Wurm, R., Raine, A., Mescher, M. \& other authors (2010). H-NS mediated repression of CRISPR-based immunity in Escherichia coli $\mathrm{K} 12$ can be relieved by the transcription activator LeuO. Mol Microbiol 77, 1380-1393.

Winstanley, C., Langille, M., Fothergill, J., Kukavica-Ibrulj, I., ParadisBleau, C., Sanschagrin, F., Thomson, N. R., Winsor, G. L., Quail, M. A. \& other authors (2009). Newly introduced genomic prophage islands are critical determinants of in vivo competitiveness in the Liverpool Epidemic Strain of Pseudomonas aeruginosa. Genome Res 19, 12-23.

Zegans, M. E., Wagner, J., Cady, K., Murphy, D., Hammond, J. \& O'Toole, G. (2009). Interaction between bacteriophage DMS3 and host CRISPR region inhibits group behaviors of Pseudomonas aeruginosa. J Bacteriol 191, 210-219.

Edited by: S. D. Bentley 